\title{
Construction and description of a constitutive plipastatin mono-producing Bacillus subtilis
}

\author{
Maliheh Vahidinasab ${ }^{1}$ Lars Lilge ${ }^{*^{*}} \mathbb{D}$, Aline Reinfurt ${ }^{1}$, Jens Pfannstiel ${ }^{2}$, Marius Henkel ${ }^{1}$, Kambiz Morabbi Heravi ${ }^{1}$ \\ and Rudolf Hausmann ${ }^{1}$
}

\begin{abstract}
Background: Plipastatin is a potent Bacillus antimicrobial lipopeptide with the prospect to replace conventional antifungal chemicals for controlling plant pathogens. However, the application of this lipopeptide has so far been investigated in a few cases, principally because of the yield in low concentration and unknown regulation of biosynthesis pathways. B. subtilis synthesizes plipastatin by a non-ribosomal peptide synthetase encoded by the $p p s A B C D E$ operon. In this study, B. subtilis 3NA (a non-sporulation strain) was engineered to gain more insights about plipastatin mono-production.
\end{abstract}

Results: The 4-phosphopantetheinyl transferase Sfp posttranslationally converts non-ribosomal peptide synthetases from inactive apoforms into their active holoforms. In case of 3NA strain, sfp gene is inactive. Accordingly, the first step was an integration of a repaired sfp version in 3NA to construct strain BMV9. Subsequently, plipastatin production was doubled after integration of a fully expressed degQ version from B. subtilis DSM10 ${ }^{\top}$ strain (strain BMV10), ensuring stimulation of DegU-P regulatory pathway that positively controls the ppsABSDE operon. Moreover, markerless substitution of the comparably weak native plipastatin promoter $\left(\mathrm{P}_{p p s}\right)$ against the strong constitutive promoter $P_{\text {veg }}$ led to approximately fivefold enhancement of plipastatin production in BMV11 compared to BMV9. Intriguingly, combination of both repaired degQ expression and promoter exchange $\left(P_{\text {pps }}: P_{\text {veg }}\right)$ did not increase the plipastatin yield. Afterwards, deletion of surfactin ( $\operatorname{srfAA-AD)}$ operon by the retaining the regulatory coms which is located within $S r f A B$ and is involved in natural competence development, resulted in the loss of plipastatin production in BMV9 and significantly decreased the plipastatin production of BMV11. We also observed that supplementation of ornithine as a precursor for plipastatin formation caused higher production of plipastatin in mono-producer strains, albeit with a modified pattern of plipastatin composition.

Conclusions: This study provides evidence that degQ stimulates the native plipastatin production. Moreover, a full plipastatin production requires surfactin synthetase or some of its components. Furthermore, as another conclusion of this study, results point towards ornithine provision being an indispensable constituent for a plipastatin monoproducer B. subtilis strain. Therefore, targeting the ornithine metabolic flux might be a promising strategy to further investigate and enhance plipastatin production by B. subtilis plipastatin mono-producer strains.

\footnotetext{
*Correspondence: lars.lilge@uni-hohenheim.de

${ }^{1}$ Institute of Food Science and Biotechnology, Department of Bioprocess

Engineering (150K), University of Hohenheim, Fruwirthstraße 12,

70599 Stuttgart, Germany

Full list of author information is available at the end of the article
}

(C) The Author(s) 2020. This article is licensed under a Creative Commons Attribution 4.0 International License, which permits use, sharing, adaptation, distribution and reproduction in any medium or format, as long as you give appropriate credit to the original author(s) and the source, provide a link to the Creative Commons licence, and indicate if changes were made. The images or other third party material in this article are included in the article's Creative Commons licence, unless indicated otherwise in a credit line to the material. If material is not included in the article's Creative Commons licence and your intended use is not permitted by statutory regulation or exceeds the permitted use, you will need to obtain permission directly from the copyright holder. To view a copy of this licence, visit http://creativeco mmons.org/licenses/by/4.0/. The Creative Commons Public Domain Dedication waiver (http://creativecommons.org/publicdomain/ zero/1.0/) applies to the data made available in this article, unless otherwise stated in a credit line to the data. 
Keywords: Bacillus subtilis, Lipopeptide, Surfactin, Fengycin, Biosurfactant, Promoter exchange, Strain engineering, Bottlenecks, Ornithine, Fungicide

\section{Background}

The preservation of food security is a global concern, especially with regard to increasing population. At the same time, there is a growing demand for organic agriculture products in both developed and developing countries. This high demand emphasizes the need for an effective, environmentally friendly alternative to chemical fertilizers, fungicides, insecticides, etc. Bacillus strains e.g. B. subtilis, B. amyloliquefaciens and B. velezensis are among the beneficial microorganisms known as effective cell factories that can produce many different secondary antimicrobial metabolites, including lipopeptides [1-3]. Plipastatin is a bioactive lipopeptide produced by Bacillus subtilis. In general, the main lipopeptides produced by Bacillus strains are classified in the three families of surfactin, iturin and fengycin $[4,5]$, including plipastatin as a member of the fengycin family [6]. Lipopeptides mostly display additional biological activities besides their amphiphilic properties. It is reported that the fengycin group exhibits a broad antagonistic effect on various soil-borne and post-harvest fungal phytopathogens, specifically on filamentous fungi $[7,8]$. Besides antifungal activity, various authors [6-10] have frequently reported the antibacterial, antiviral and anticancer properties of fengycins. Additionally, these lipopeptides act also as the elicitor of induced systemic resistance in plants [11]. However, in contrast to the very well investigated applicability of surfactin, the application potential of fengycins have so far been investigated only in a few cases, principally based on poor bacterial productivity of fengycins and therefore, laborious and ineffective production approaches.

The structure of lipopeptides comprises a fatty acid connected to a peptide moiety [12]. The composition of amino acids in the circular peptide chain and length of the fatty acid residue provides a unique property for every lipopeptide [13]. Plipastatin consists of a decapeptide chain (L-Glu - D-Orn - L-Tyr - D-Thr - L-Glu - D-Ala - L-Pro -L-Gln - D-Tyr - L-Ile), 8 amino acids of which form a peptide ring and is linked to a 3-hydroxy fatty acid with 14-19 carbon atoms that may be saturated or unsaturated $[14,15]$. Although, the structure of fengycin is almost identical to plipastatin, the enantiomers of the amino acids in positions 3 and 9 (Tyr3 and Tyr9) are present in the L- and D-form in plipastatin, respectively, while in fengycin, they are in the reverse configuration $[6,13,16]$. Plipastatin and other lipopeptides are formed by step-by-step reactions of specific non-ribosomal peptide synthetases (NRPSs) [17]. The lipopeptide biosynthesis depends on the 4-phosphopantetheinyl transferase Sfp which converts the inactive apoforms of NRPSs to the active holoforms [18]. In case of $B$. subtilis 168 , the genome has two large operons of $\operatorname{srfAA} A D$ and ppsABCDE, which encode the subunits of NRPSs for surfactin and plipastatin production, respectively. However, due to a single base duplication in sfp gene, B. subtilis 168 is incapable to synthesize these lipopeptides [19-21]. Nevertheless, after repairing the $s f p$ mutation, its lipopeptide production is restored [22, 23].

Usually, Bacillus spp. encode for more than one lipopeptide synthetase, which are not synthesized simultaneously in the same growth phase showing the involvement of different regulators. More specifically, in the genome of bacteria that have the ppsAB$C D E$ operon, the $\operatorname{srfAA-AD}$ operon is always present as well $[24,25]$. However, while surfactin is being produced in the late exponential phase, the biosynthesis of plipastatin has been characterized in the stationary phase [25-27]. Lipopeptide synthetases are usually regulated by complex regulatory networks in a growthphase dependent manner. In the case of plipastatin, the pps $A B C D E$ operon is repressed by $\mathrm{AbrB}$, the transition state regulator, during exponential growth phase [28]. Moreover, expression of the pleiotropic regulator $\operatorname{deg} Q$ gene has a positive effect on the synthesis of plipastatin showing the possible involvement of DegSU twocomponent system $[20,21,29]$. In B. subtilis 168 , the -10 promoter region of $\operatorname{deg} Q$ has a single base mutation that leads to low gene expression. Nevertheless, substitution of this mutation enables overexpression of the $\operatorname{deg} Q$ gene and results in increased plipastatin biosynthesis [23, 30, 31].

In this study, we used B. subtilis $3 \mathrm{NA}$ as a model strain for genetic engineering of plipastatin production in order to construct a plipastatin mono-producer. This strain was previously described as a hybrid strain encoding genetic features from $B$. subtilis W23 and 168 [21]. In detail, the surfactin $(\operatorname{srf} A A-A D)$ operon was deleted in a way that their competence formation remained intact. Subsequently, the plipastatin was increased by promoter exchange and manipulation of the $\operatorname{deg} Q$ expression. Finally, the effect of supplementing potentially critical amino acids on plipastatin formation was evaluated. 


\section{Results}

\section{Plipastatin production in different $B$. subtilis strains}

To construct a plipastatin mono-producer, two B. subtilis strains were compared for their potential in biosynthesis of plipastatin. This included the derivatives of the wellknown laboratory model strain B. subtilis 168 and strain 3NA. Plipastatin operon and its promoter region are identical in these two strains [21]. Nevertheless, strain 3NA is a sporulation-deficient strain caused by a frame shift mutation in $s p o 0 A$ which makes this strain suitable for fermentation [21]. These domesticated B. subtilis strains are known to have a mutation in their $s f p$ gene disabling them to produce lipopeptides, such as surfactin and plipastatin $[20,21]$. Therefore, the $s f p+$ derivates of strain 168 (JABs24) and strain 3NA (BMV9) were used to ensure lipopeptide production. Both strains were cultivated in mineral salt medium. Figure 1 shows the lipopeptide production, glucose consumption and optical density $\left(\mathrm{OD}_{600}\right)$ over $72 \mathrm{~h}$ of cultivation at $30{ }^{\circ} \mathrm{C}$. The comparison of growth rates showed a faster cell growth of BMV9 strain compared to JABs24. However, optical density of BMV9 was drastically decreased without any stationary phase after glucose depletion. In contrast,

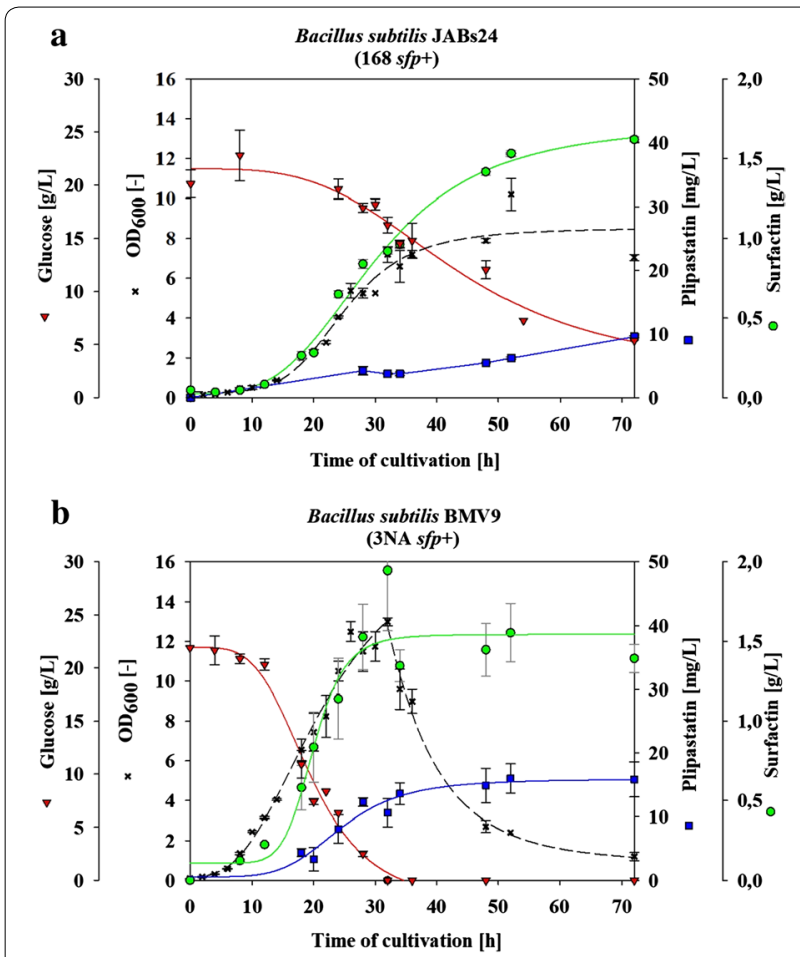

Fig. 1 Comparison of cultivation parameters for a $B$. subtilis JABs24 $(168 s f p+)$ and $\mathbf{b}$ B. subtilis BMV9 (3NA sfp +); The shake flask cultivations were performed in mineral salt medium containing $20 \mathrm{~g} / \mathrm{L}$ glucose and $50 \mathrm{mM}$ urea as carbon and nitrogen sources at $30{ }^{\circ} \mathrm{C}$ and $120 \mathrm{rpm}$. Besides optical density $\left(\mathrm{OD}_{600}\right)$, glucose consumption, plipastatin and surfactin production were detected
JABs24 exhibited an entry into the stationary phase when $20 \%$ of glucose were metabolized. Both strains revealed surfactin production from the beginning of cultivation through to the early stage of stationary phase. Thus, surfactin quantities gradually increased until a peak was reached in the late exponential phase. In case of plipastatin, no detectable amounts could be measured up to the middle of exponential phase. The highest amount of plipastatin was produced by BMV9 in the stationary phase and was about $17 \mathrm{mg} / \mathrm{L}$. Conversely, the production of plipastatin by JABs24 was lower than BMV9 $(10 \mathrm{mg} / \mathrm{L}$ after $72 \mathrm{~h}$ ). Based on these results strain BMV9 was considered for further strain engineering.

\section{Verification of different putative bottlenecks concerning plipastatin production}

As mentioned before, B. subtilis $3 \mathrm{NA}$ is a hybrid strain that exhibits genetic features from both strains W23 and 168 [21]. Likewise, the $\operatorname{deg} Q$ gene and upstream promoter region are identical to that of strain 168. Therefore, a single base mutation in the $\operatorname{deg} Q$ promoter region drastically decreases the corresponding gene expression [32]. Previous studies demonstrated that this circumstance has a negative effect on plipastatin production [31]. We hypothesized that a repair of $\operatorname{deg} Q$ expression combined with the deletion of the competitive surfactin operon as well as an exchange of a weak plipastatin promoter against a constitutively active promoter lead to a highly efficient plipastatin mono-producer strain. However, before constructing the mutant strain that exhibits these three characteristics, BMV9 strain (3NA $s f p+$ ) was used to construct BMV10, BMV11 and BMV12, showing repaired $\operatorname{deg} Q$ expression, plipastatin promoter exchange and surfactin elimination, respectively. Based on the observation that plipastatin level reached a plateau after $40 \mathrm{~h}$ of cultivation (Fig. 1), the production outcomes of all following mutant strains were measured after $48 \mathrm{~h}$ of cultivation (Fig. 2).

As shown in Fig. 2, BMV10 which holds a repaired $\operatorname{deg} Q$ gene, showed a twofold higher plipastatin production but a decrease in surfactin formation compared to the control strain (BMV9). Furthermore, the promoter exchange of native plipastatin promoter against the constitutive promoter region of the veg gene resulting in BMV11 strain, increased the plipastatin titer from $15 \mathrm{mg} / \mathrm{L}$ in control strain BMV9 to $70 \mathrm{mg} / \mathrm{L}$. Interestingly, the surfactin formation was unaffected by the enhanced plipastatin production. In order to eliminate surfactin synthesis, the entire $\operatorname{srf} A A-A D$ operon was deleted and the regulatory com $S$ gene which is co-expressed with $\operatorname{srf} A A-A D$ operon restored back to the genome with the native $\mathrm{P}_{s r f A}$ promoter. Consequently, the strains BMV12 $\left(\triangle s r f A A-s r f A D\right.$ with native $\mathrm{P}_{p p s}$ promoter) and BMV13 


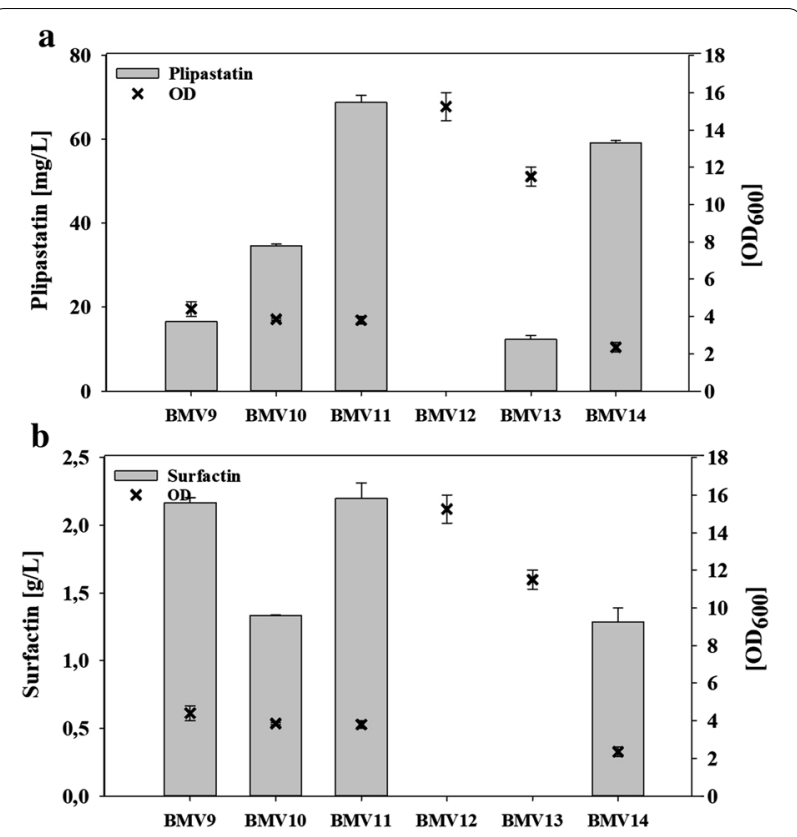

Fig. 2 Overview about production of plipastatin (a) and surfactin (b) in respect to reached optical densities $\left(\mathrm{OD}_{600}\right)$ of different engineered mutant strains after $48 \mathrm{~h}$ cultivation in mineral salt medium; BMV9, control strain (3NA sfp +); BMV10, repaired degQ expression; BMV11, promoter exchange of pps operon; BMV12, deletion of srfA operon; BMV13, combination of promoter exchange and srfA operon deletion; BMV14, combination of promoter exchange and repaired degQ expression

( $\triangle$ srfAA-srfAD with $\mathrm{P}_{p p s}:: \mathrm{P}_{\text {veg }}$ promoter exchange) were constructed. It was observed that even though the deletion of the surfactin operon caused enhanced cell growth in both BMV12 and BMV13 strains, plipastatin production reduced (Fig. 2a). Plipastatin production was significantly decreased not only under native expression of plipastatin operon (BMV12) but also when $\mathrm{P}_{p p s}$ was exchanged against constitutive $\mathrm{P}_{v e g}$ promoter (BMV13).

In order to see the effect of combining the features of promoter exchange and repairing $\operatorname{deg} Q$ gene expression, the strain BMV14 was constructed. Comparably to strain BMV9, the expression of $\operatorname{deg} Q$ had a negative effect on surfactin production (Fig. 2b). Interestingly, no additive effect was observed on plipastatin production by BMV14 (Fig. 2a). In summary, we concluded that under the same conditions, BMV11 was able to produce the highest amounts of plipastatin compared to all other mutant strains constructed.

\section{Impact of amino acid supplementation on plipastatin production}

To verify the impact of amino acid precursors on plipastatin and surfactin formation (Additional file 1), seven different amino acids including glutamic acid, glutamine,
Table 1 Plipastatin titers achieved of B. subtilis BMV9 (3NA $s f p+)$ and B. subtilis BMV12 (3NA sfp + plipastatin monoproducer)

\begin{tabular}{lcl}
\hline Supplementation & $\begin{array}{l}\text { B. subtilis BMV9 } \\
\text { Plipastatin (mg/L) }\end{array}$ & $\begin{array}{l}\text { B. subtilis BMV12 } \\
\text { Plipastatin (mg/L) }\end{array}$ \\
\hline Control & $19.1 \pm 0.1$ & n. d. \\
Glutamic acid & $7.8 \pm 0.2$ & n. d \\
Glutamine & $6.6 \pm 0.9$ & n. d \\
Isoleucine & $10.5 \pm 2.6$ & n. d \\
Alanine & $14.3 \pm 0.9$ & n. d \\
Threonine & $11.3 \pm 0.8$ & n. d \\
Proline & $14.4 \pm 1.3$ & n. d \\
Ornithine & $18.4 \pm 2.4$ & $6.3 \pm 0.5$ \\
\hline
\end{tabular}

Plipastatin titers measured after $48 \mathrm{~h}$ cultivation in mineral salt medium supplemented with $30 \mathrm{mM}$ of the indicated amino acids compared to control cultivations (without any amino acid supplementation).

*Not detectable

isoleucine, alanine, threonine, proline and ornithine with a concentration of $30 \mathrm{mM}$ were additionally supplemented in mineral salt medium. BMV9 (3NA $s f p+)$ and BMV12 (3NA $s f p+$ plipastatin mono-producer) were selected to evaluate the produced plipastatin under control of native $\mathrm{P}_{p p s}$ promoter, in the presence and absence of the surfactin operon.

As it is shown in Table 1, except for ornithine, a decrease in plipastatin titer was observed when supplementing BMV9 strain with the other six amino acids. Interestingly, in the plipastatin mono-producer BMV12 strain, supplementation of the ornithine led to a detectable plipastatin titer. Neither in control cultures (without amino acid supplementation) nor in the presence of other amino acids, no detectable production was observed. Subsequently, it was observed that cultivation of BMV13 (constitutive plipastatin mono-producer) in supplemented medium with ornithine let to enhance in plipastatin titer (about 10\%). Furthermore, it is noteworthy to mention that the observed plipastatin chromatogram on silica HPTLC plate of BMV12 (plipastatin mono-producer) in the presence of ornithine exhibited a modified pattern compared to the plipastatin standard (B. subtilis plipastatin/fengycin standard, Lipofabrik france) and parental BMV9 strain (Fig. 3A). Although the detected signals in the chromatogram showed similar $\mathrm{Rf}$ values compared to the standard, not all standard peaks could be observed. Therefore, HPLC-MS analysis was performed for identification of plipastatin variants or homologs. 
(See figure on next page.)

Fig. 3 A Detected HPTLC chromatograms of the standard plipastatin in comparison with plipastatin produced by BMV9 (3NA sfp +) and BMV12 (3NA plipastatin mono-producer) with and without ornithine supplementation. Plipastatin standard produced by B. subtilis (Lipofabrik, France) [a], plipastatin produced by BMV9 strain after $48 \mathrm{~h}$ cultivation in mineral salt medium without any amino acid and with ornithine supplementation [b]; plipastatin produced by BMV12 strain after $48 \mathrm{~h}$ cultivation in mineral salt medium without amino acid and with ornithine supplementation [c]. B Comparison of plipastatin variants ratios in plipastatin standard and mono-producer BMV12 strain during exponential and stationary phase. The BMV12 was cultivated in mineral salt medium supplemented with $30 \mathrm{mM}$ of ornithine. The samples were taken after $24 \mathrm{~h}$ (exponential phase) and $48 \mathrm{~h}$ (stationary phase). Peak areas were determined by extracted ion chromatograms for each plipastatin variant using their precise $\mathrm{m} / \mathrm{z}$ values. Ratios of the peak areas of plipastatin variants were calculated within the standard and the two sample points

\section{Mass spectrometry analyses of plipastatin variants produced by BMV12 with supplementation of ornithine}

Plipastatin produced by $B$. subtilis is a mixture of several homologs based on the length of fatty acid chain and variants within peptide moiety [33]. The patterns of the HPTLC chromatograms of produced plipastatin are comparable between the $B$. subtilis plipastatin standard and the sample extracts of the parental BMV9 strain. In both cases, plipastatin splits into several peaks. This might indicate that several plipastatin variants were produced (Fig. 3A [a, b]). Subsequently, plipastatin pattern of mono-producer strain BMV12 was analyzed by HPTLC and it appeared in one peak in the same Rf values (Fig. 3A [c]). Thus, it was assumed that deletion of $\operatorname{srfA}$ operon resulted in an accumulation of specific plipastatin variants whereas other subtypes were diminished. To get a perception about the different plipastatin variants present in B. subtilis, the high-purified plipastatin standard was analyzed by mass spectrometry (HPLC-ESI-MS). The results showed that a large variety of plipastatin subtypes with saturated and unsaturated fatty acid chains is present in the plipastatin standard (Additional file 2A). Mainly, A and B type plipastatin were detected in the LC-ESI-MS analysis, but also other plipastatin variants were observed. Some of them could be assigned to plipastatin C, D or S types [34] based on their diagnostic marker ions. In addition, other plipastatin variants were detected that were not described in previous studies in $B$. subtilis so far. Therefore, they were named in this study as $\mathrm{W}, \mathrm{U}$ and $\mathrm{Y}$. To get an overview about the differences in plipastatin variants produced by BMV12, shake flask cultivation was performed in mineral salt medium supplemented with ornithine. Samples were then harvested from the exponential and stationary phase (plipastatin HPTLC chromatograms were comparable in both growth phases) and also analyzed by LC-ESI-MS. Afterwards, the peak areas of identified plipastatin variants were used to determine ratios in between BMV12 samples and were compared to that of the plipastatin standard (Fig. 3B). Variants of the subtypes plipastatin A and plipastatin B were detected in both BMV12 samples and the standard, even though especially plipastatin $\mathrm{B} \mathrm{C}_{14} \mathrm{u}$ (unsaturated fatty acid chain with 14 carbon atoms) and plipastatin
$\mathrm{B} \mathrm{C}_{15} \mathrm{~s}$ (saturated fatty acid chain with 15 carbon atoms) showed higher relative abundance in the standard in contrast to BMV12 samples. In contrast, slightly higher abundances of plipastatin $\mathrm{B} \mathrm{C}_{16} \mathrm{u}$ and plipastatin $\mathrm{B}$ $\mathrm{C}_{18} \mathrm{~s}$ were detected in both samples of BMV12. Taken together, no major differences were detected between standard and BMV12 plipastatin extracts. Hence, the reason for the variation of the plipastatin pattern based on the deletion of surfactin operon could not be explained. Another interesting observation was the accumulation of different plipastatin variants (named in this study plipastatin $\mathrm{Y}, \mathrm{W}$ and $\mathrm{U}$ ) during the stationary phase. Due to the cultivation with mineral salt medium, a limitation of the amino acids during the stationary phase is very likely. Insofar, the accumulation of plipastatin $\mathrm{Y}, \mathrm{W}$ and $\mathrm{U}$ could be due to the incorporation of different amino acids into the peptide residue of plipastatin.

\section{Discussion}

In this study, B. subtilis 3NA was engineered to construct a plipastatin mono-producer strain and to gain more insights about plipastatin production. Previously, it was shown that DegQ positively regulates plipastatin production [30]. In fact, DegQ stimulates autophosphorylation of DegS sensor kinase resulting in enhanced phosphotransfer to DegU response regulator [29, 35]. As a result, the phosphorylated and activated DegU-P version causes higher expression of pps $A B C D E$ operon and increases the plipastatin production [36]. In this study, repair of $\operatorname{deg} Q$ expression (strain BMV10) ensured DegU-P activation, which doubled the production of plipastatin compared to parental BMV9 strain (3NA $s f p+$ ). In this context, previous results from Tsuge et al. [30] showed a tenfold higher plipastatin production when $p p s A B C D E, \operatorname{deg} Q$ and $s f p$ were combined in a $B$. subtilis plasmid expression system. Furthermore, Wang et al [31]. described a decrease of plipastatin after in-frame mutagenesis of $\operatorname{deg} Q$ in $B$. subtilis NCD-2. Afterwards, with respect to the relatively low expression level of ppsABCDE operon, an approximately fivefold higher plipastatin formation was achieved by the exchange of the native $\mathrm{P}_{p p s}$ promoter against constitutive $\mathrm{P}_{\text {veg }}$ promoter (strain BMV11). Previous to this study, the effect of promoter exchange on the amount of plipastatin 
A

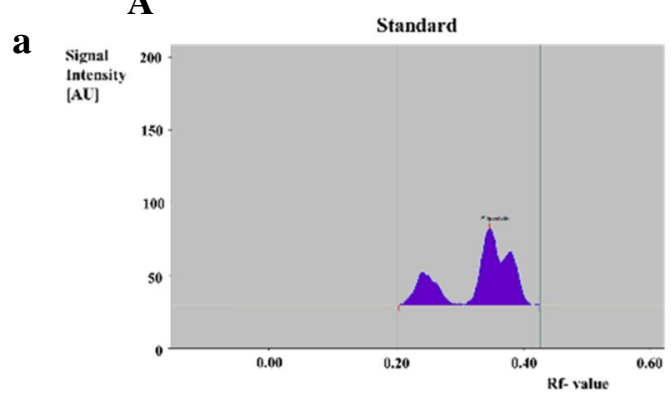

b
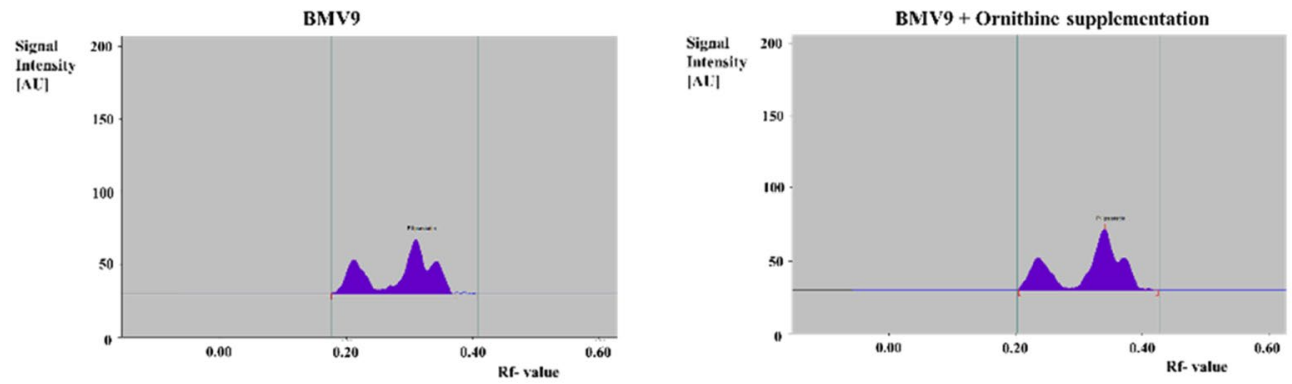

C
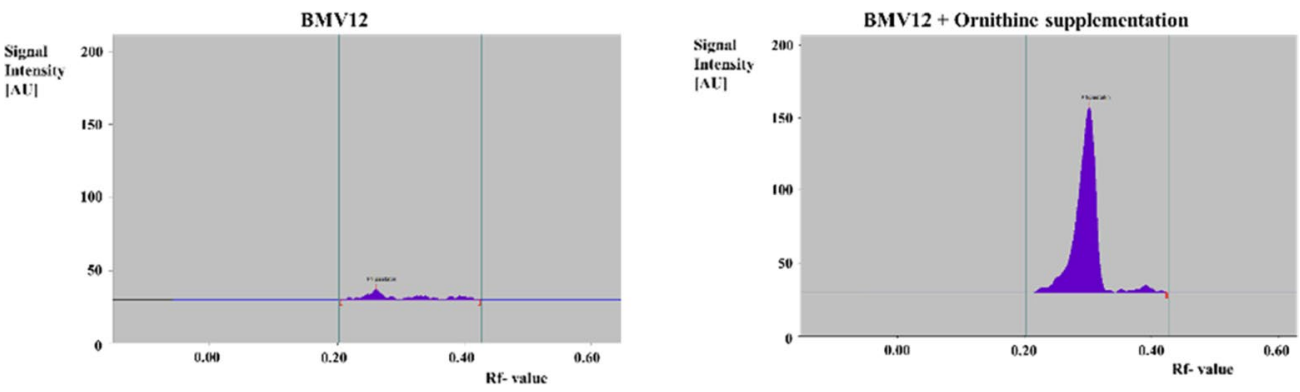

B
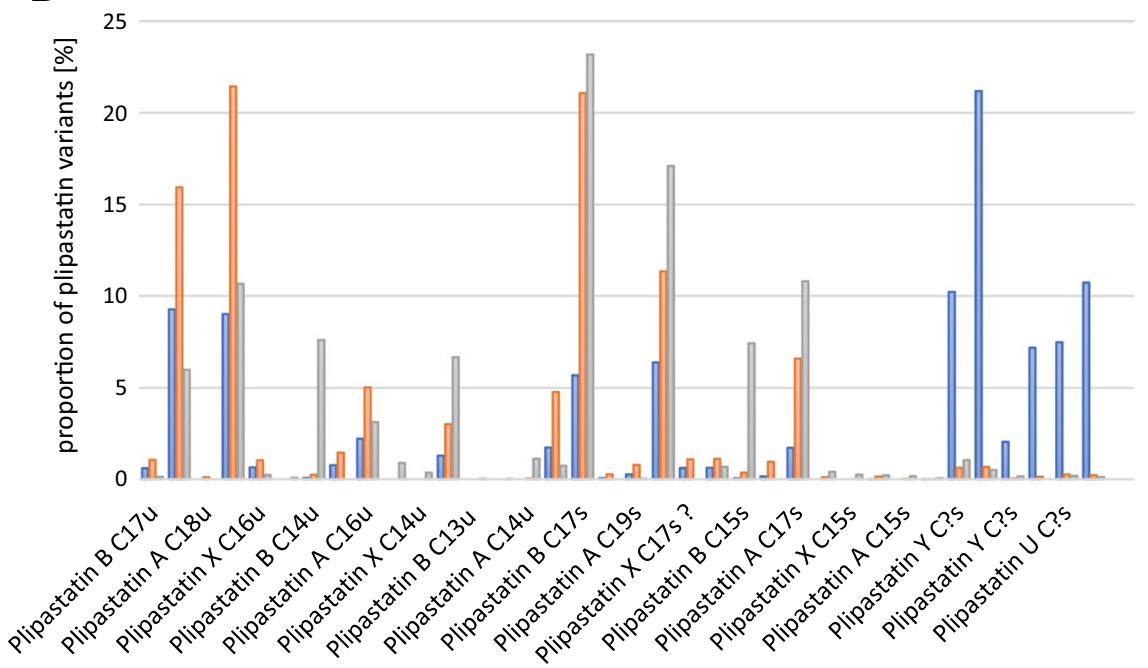

$\square$ BMV12 stationary phase

$\square$ BMV12 exponential phase

$\square$ Standard 
produced was reported only on a few cases. For instance, promoter exchange of native $\mathrm{P}_{p p s}$ promoter against native $\mathrm{P}_{\text {fen }}$ from $B$. subtilis 21332 caused no plipastatin overproduction. In contrast, a tenfold higher plipastatin production was obtained when $\mathrm{P}_{\text {fen }}$ from strain BBG21 (a spontaneous mutant of B. subtilis ATCC 21332) was integrated [37]. In the following step, it was observed that the combination of both repair of $\operatorname{deg} Q$ expression and the $\mathrm{P}_{p p s}$ promoter exchange (BMV14), had no additional effect on the plipastatin titer compared to BMV11 (constitutive plipastatin producer). This is in contrast to the comparison of parental BMV9 and BMV10 when functional $\operatorname{deg} Q$ expression increases plipastatin production about twofold. An explanation could be addressed by the DegQ mediated activation of DegU-P regulon, which causes in general the increase of secretory proteases [38, 39]. These proteases could target plipastatin for degradation. In sum, this negative effect could be more noticeable in a constitutive pps operon expression $\left(\mathrm{P}_{v e g}\right)$ which make a higher amount of DegQ not benefical.

Another aim of this study was to construct a strain that ensures constitutive plipastatin mono-production. In a recent work, it was shown that deletion of $\operatorname{srfAA}$ significantly reduced the plipastatin production and on the other hand deletion of $\operatorname{srfAC}$ showed no effect [25]. The authors argued that probably $\operatorname{srfAA}$ has a regulatory effect on plipastatin production. Furthermore, they also observed another significant decrease in plipastatin production when pnp gene, which is responsible for biosynthesis of multifunctional polynucleotide phosphorylase (PNPase) [40] was deleted. The regulation of PNPase on plipastatin biosynthesis can happen through effect on comS expression. Subsequently, it was hypothesized that coms expression in an unknown complex pathway positively regulates plipastatin formation. In this study, we have deleted the whole $\operatorname{srf} A A-A D$ operon and retained comS with the native $\mathrm{P}_{\text {srfA }}$ promoter (BMV12). In BMV12, even though comS was retained, plipastatin titer decreased to a non-detectable concentration confirming previously described study [25] that $\operatorname{srfAA}$ or in general, the expression of surfactin synthetase has a positive effect on plipastatin production. This observation was consistent after exchange of native $\mathrm{P}_{p p s}$ against $\mathrm{P}_{\text {veg }}$ (BMV11 compared to BMV13). Likewise, in another study $\mathrm{P}_{p p s}$ promoter was exchanged against a strong $\mathrm{P}_{\text {amy }}$ promoter from Bacillus amyloliquefaciens resulting in an increase in plipastatin production. However, after elimination of surfactin synthetase by deletion of $\operatorname{srfAB}, \operatorname{srf} A C$ and $\operatorname{srf} A D$ genes, plipastatin production did not change [11]. Hence, in respect to our results and previously described studies $[11,25]$ we conclude that, subunits of the surfactin synthetase have different impacts on plipastatin production.
Another rational factor in increasing the plipastatin production is the presence of a sufficient quantity of precursors in the cultivation medium. Therefore, we have attempted to improve plipastatin production by supply of seven amino acids of the plipastatin peptide chain in the mineral salt medium. Previously, these seven amino acids as the only nitrogen source were used in the mineral salt medium and compared with other nitrogen sources such as urea and ammonium carbonate [41]. Accordingly, urea was introduced as the best nitrogen source and alanine, followed by glutamic acid, were the best sources of nitrogen among the other amino acids. In this study, approximately the same concentration of amino acids were added to the medium besides urea as the main nitrogen source. Since, surfactin synthetase, assumably has a regulatory effect on plipastatin production, the supplementation of amino acids was examined in two strains of BMV9 (3NA $s f p+$ ) and BMV12 (3NA $s f p+$ plipastatin mono-producer). Among all the amino acids used, ornithine was the only amino acid induced a detectable plipastatin production in mono-producer (BMV12), while it has not been measured in the other cultivations of BMV12. Interestingly, in the strain BMV9, the supplementation of different amino acids had different effects on final plipastatin titer. As it was shown in Fig. 1, the optical density of BMV9 directly decreased after glucose consumption whereas the plipastatin titer remained stable over time. Therefore, a comparison of the plipastatin production per biomass after $48 \mathrm{~h}$ cultivation is not reasonable. Accordingly, the results show that expect for ornithine, the additional supplementation of amino acids reduced in general the plipastatin titer in BMV9. This can be explained due to (de-) activation of stringent response in B. subtilis which occurs in amino acid limitation. Activation of stringent response results in enhanced provision of branched-chain amino acids [42, 43]. Conversely, by addition of amino acids in the cultivation medium, the positive side effect of stringent response will be reduced. However, supplementation of ornithine in the medium had no negative effect and it was the only amino acid that enhanced the plipastatin produced by BMV12 (plipastatin mono-producer) to a detectable level. Therefore, it is concluded that ornithine is an indispensable constituent for a plipastatin mono-producer B. subtilis strain.

\section{Conclusions}

This study provides evidence that $\operatorname{deg} Q$ stimulates the native plipastatin production. A significant decrease in plipastatin productivity after deletion of the surfactin operon in a constitutive plipastatin producer strain suggested that full plipastatin production requires the surfactin synthetase or some of its components. Nevertheless, the impact of surfactin synthetase existence on 
Table 2 Bacterial strains and plasmids used in this study

\begin{tabular}{|c|c|c|c|}
\hline Strain or plasmid & \multicolumn{2}{|l|}{ Genotype or description } & Reference \\
\hline \multicolumn{4}{|l|}{ Strains } \\
\hline \multicolumn{4}{|l|}{ Escherichia coli } \\
\hline JM109 & \multicolumn{2}{|c|}{ mcrA recA1 supE44 endA1 hsdR17 $\left(r_{K}^{-} m_{K}^{+}\right)$gyrA96 relA1 thi $\Delta\left(\right.$ lac-proAB) $F^{\prime}\left[\right.$ traD36 proAB ${ }^{+}$lacla lacZ M15] } & [48] \\
\hline \multicolumn{4}{|l|}{ Bacillus subtilis } \\
\hline JABs24 & \multicolumn{2}{|l|}{ B. subtilis $168 \triangle m a n P A ; \operatorname{trp}+;$ sfp + ; } & [49] \\
\hline 3NA & & {$[50]$} \\
\hline JABs32 & \multicolumn{2}{|l|}{ spo0A3; $\triangle$ manPA::erm; sfp +; } & $\begin{array}{l}\text { J. Altenbuch- } \\
\text { ner (unpub- } \\
\text { lished) }\end{array}$ \\
\hline BMV9 & \multicolumn{2}{|l|}{ spo0A3; $\triangle m a n P A ; s f p+;$} & This study \\
\hline BMV10 & \multicolumn{2}{|l|}{ spoOA3; $\triangle m a n P A ;$ sfp + ; $\triangle a m y E:: \operatorname{deg} Q$ (from B. subtilis DSM10 ${ }^{\top}$ ) } & This study \\
\hline BMV11 & \multicolumn{2}{|l|}{ spoOA3; $\triangle m a n P A ; s f p+; P_{p p s}-p p s A-E:: P_{\text {veg }}-p p s A-E$} & This study \\
\hline BMV12 & \multicolumn{2}{|l|}{ spo0A3; $\triangle m a n P A ; s f p+; \triangle s r f A A-A D::$ coms-erm } & This study \\
\hline BMV13 & \multicolumn{2}{|c|}{ spoOA3 $\triangle m a n P A ; s f p+; \triangle s r f A A-A D:: c o m S-e r m ; P_{p p s}-p p s A-E:: P_{\text {veg }}-p p s A B C D E$} & This study \\
\hline BMV14 & \multicolumn{2}{|c|}{ spo0A3; $\triangle m a n P A ; s f p+; P_{p p s}-p p s A B C D E:: P_{\text {veg }}-p p s A B C D E ; \triangle a m y E:: \operatorname{deg} Q$ (from B. subtilis DSM10 ${ }^{\top}$ ) } & This study \\
\hline \multicolumn{4}{|c|}{ 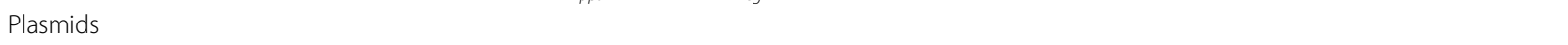 } \\
\hline pJOE6743.1 & \multicolumn{2}{|l|}{ ori $_{\text {puc18, }}$ bla, spc, manP, ter-lacl-lacZa-ter } & [51] \\
\hline pJOE7644.2 & \multicolumn{2}{|l|}{ ori $_{\text {puc18 }}$, bla, $\mathrm{P}_{\text {manp }}-$ manP, spc, manR-ctaO } & [46] \\
\hline pJOE4786.1 & \multicolumn{2}{|l|}{ ori $_{\text {puc18, }}$ bla, ter-'lacl-lacZa-ter } & [52] \\
\hline pKAM312 & \multicolumn{2}{|c|}{ ori $_{\text {pBR322, }}$ rop, ermC, bla, amyE'-[ter-P glcR - lacZ-spcR]-'amyE } & [46] \\
\hline PMAV3 & \multicolumn{2}{|c|}{$\begin{array}{ll}\text { pJOE4786.1 containig } P_{\text {veg }}:: P_{p p s} \text { exchange fragment (integrated by Sma I) } & \text { This study } \\
\text { J.OE6743. containig } P \text {.P. }\end{array}$} & \\
\hline pMAV4 & pJOE6743.1 containig $\mathrm{P}_{\text {veg }}: \mathrm{P}_{\text {pps }}$ exchange fragment (integrated by Hind III) & This study & \\
\hline pMAV5 & \multicolumn{2}{|l|}{ pKAM312 containing $\operatorname{deg} Q\left(B\right.$. subtilis DSM10 $0^{\top}$ ) (integrated by Hind III) } & This study \\
\hline
\end{tabular}

plipastatin formation is still unknown. In order to construct a plipastatin mono-producer strain suitable for cultivation in large quantities in a bioreactor, understanding the mutual impact between surfactin and plipastatin syntheses might help to increase the final plipastatin production. Furthermore, as another conclusion of this study, results point towards ornithine provision being an indispensable constituent for a plipastatin mono-producer B. subtilis strain. Therefore, targeting the ornithine metabolic flux might be a promising strategy to further investigate and enhance plipastatin production by B. subtilis plipastatin mono-producer strains.

\section{Materials and methods}

\section{Chemicals, materials and standard procedures}

All chemicals were acquired from Carl Roth $\mathrm{GmbH} \&$ Co. KG (Karlsruhe, Germany) if not, mentioned otherwise. Standard molecular techniques were carried out as described by Sambrook and Russell [44]. The desired DNA fragments were amplified in polymerase chain reactions using DNA Polymerase (Phusion High-Fidelity \#M0530S, New England BioLabs, Frankfurt am Main, Germany). The PCRs were carried out on a PCR thermal cycler (prqSTAR 96X VWR GmbH, Darmstadt, Germany). Chromosomal DNA was purified with a ready to use kit (innuPREP Bacteria DNA Kit) and plasmid DNA was extracted with innuPREP Plasmid Mini Kit (Analytik Jena AG, Jena, Germany). After PCR reactions, amplified DNA fragments were extracted after agarose-based gel electrophoresis with QIAquick PCR \& Gel Cleanup Kit, according to the manufactures' instruction. Restriction enzymes and alkaline phosphatase (\#M0290) was purchased from New England BioLabs (Frankfurt am Main, Germany) and T4 DNA ligase were purchased from Thermo Fisher Scientific (Karlsruhe, Germany). All ligation reactions were performed overnight at $4{ }^{\circ} \mathrm{C}$. For better efficiency of ligation, a PEG 8000 solution was added. Oligonucleotides were synthesized by Eurofins MWG (Ebersberg, Germany).

\section{Strains, plasmids and transformation method}

All strains and plasmids used in this study are shown in Table 2. Oligonucleotides used for construction of strains and plasmids are listed in Table 3. Escherichia coli JM109 was used for plasmid propagation and cloning. Transformation of $E$. coli strains were carried out according to the standard heat-shock method [45]. B. subtilis JABs32 strain, a $s f p$ + derivate of $B$. subtilis $3 \mathrm{NA}$, was used for mannose counterselection. Therefore, erythromycin resistance gene $(\mathrm{erm})$ for $\operatorname{manPA}$ deletion was removed 
Table 3 Oligonucleotides used in this study

\begin{tabular}{|c|c|c|}
\hline Name & Sequence $5^{\prime}-3^{\prime}$ & Purpose \\
\hline s1009 & CTGCCGTTATTCGCTGGATT & \multirow{4}{*}{$\begin{array}{l}\left.\text { Integration of degQ gene ( } B \text {. subtilis } \mathrm{DSM} 10^{\top}\right)(+510 \mathrm{bp}) \text { in amyE locus } \\
\text { underlined sequences highlight the } N d e \text { and EcoR I restriction site }\end{array}$} \\
\hline s1410 & ATTATTAACATATGCGGCGTACCTCATACGGATACAC & \\
\hline s1409 & ATTATTAAGAATTCCTCCTTGATCCGGACAGAATC & \\
\hline s1010 & AGAGAACCGCTTAAGCCCGA & \\
\hline s1221 & GGAAAGTGAAAAAAGGAGAAGG & \multirow[t]{6}{*}{ Construction of $\mathrm{P}_{\text {veg }}:: \mathrm{P}_{p p s}$ promoter exchange } \\
\hline s1222 & CCTATGCAGGTTTTCAACTGTTATTGATTTGCCAAAATGACAG & \\
\hline s1223 & CAGTTGAAAACCTGCATAGG & \\
\hline s1224 & TGCATCCACCTCACTACAT & \\
\hline s1225 & ATGTAGTGAGGTGGATGCATTGAGCGAACATACTTATTCTTTAAC & \\
\hline s1226 & CATTTAAAGAGATTCCATCCATTATGATATG & \\
\hline s1162 & CATGATTTTCAGGTCTGCAAGAAC & \multirow[t]{8}{*}{ Construction of srfAA-AD:: comS-erm } \\
\hline s1163 & GTTCAAACGTCTGCTCCTCCTTAATCTTTATAAGCAGTGAACATGTGC & \\
\hline s1164 & AGGAGGAGCAGACGTTTGAAC & \\
\hline s1165 & CTTCTCCCTCCAGCAGAAGTAC & \\
\hline s1166 & $\begin{array}{l}\text { CTTCTGCTGGAGGGAGAAGTAGGTATAAATTTAAC-GATCACTCATCA } \\
\text { TGTTC }\end{array}$ & \\
\hline s1167 & GACCGATAGATTTTGAATTTAGGTGTC & \\
\hline s1168 & CACCTAAATTCAAAATCTATCGGTCGAATGCCAAT-TTCTGCATGGTATAATAG & \\
\hline s1169 & GGCAACCTGATGGATAAAGAAATTG & \\
\hline
\end{tabular}

by the use of plasmid pJOE7644.2 resulting in BMV9 [46]. Strain BMV9 was used as parental strain for construction of further mutant strains. Transformation of natural competent B. subtilis strains was performed according to the "Paris method" [47]. Depending on the selection marker, the transformants were selected on LB agar supplemented with ampicillin $(100 \mu \mathrm{g} / \mathrm{mL})$, spectinomycin $(100 \mu \mathrm{g} / \mathrm{mL})$ or erythromycin $(10 \mu \mathrm{g} / \mathrm{mL}$ for E. coli and $5 \mu \mathrm{g} / \mathrm{mL}$ for B. subtilis). All plates were incubated at $37^{\circ} \mathrm{C}$.

\section{Construction of plasmids for strain engineering}

For markerless promoter exchange, LFH-PCR method was used [53]. Accordingly, upstream and downstream wild-type sequence of pps promoter region was fused with veg promoter. After ligation into Sma I digested pJOE4786.1 resulting in pMAV3, target sequence was isolated by Hind III digestion and was subsequently integrated into pJOE6743.1 (results in pMAV4). Afterwards, plasmid pMAV4 was transformed into strain BMV9 followed by the protocol described before [51].

For the integration of the $\operatorname{deg} Q$ gene including promoter region $(+510 \mathrm{bp})$ and terminator structure from B. subtilis DSM10 ${ }^{\mathrm{T}}$, the primer $\mathrm{s} 1410$ and $\mathrm{s} 1409$, containing Nde I and EcoR I restriction sites, were used. After restriction digestion, the $\operatorname{deg} Q$ fragment was ligated into pKAM312 [46] resulting in pMAV5. After transformation of pMAV5 into parental strain BMV9 and other mutant strains, transformants were selected on LB agar plates containing spectinomycin. To ensure the correctness of plasmids and mutant strains, all constructs were confirmed by sequencing (Eurofins Genomics Germany GmbH, Ebersberg, Germany).

\section{Deletion of surfactin operon and retain of coms gene}

The principle of LFH-PCR was utilized to design a DNA fragment for deletion of $\operatorname{srf} A$ operon and simultaneously retain of comS gene. A fusion of upstream region of $\operatorname{srfA}$ operon with comS gene ensured a wild-type expression. For a simple strain selection, com $S$ gene was additionally linked with erythromycin resistance cassette (erm) of pKAM312. An uncoupled erm gene expression was ensured by maintaining the natural $\mathrm{P}_{\text {erm }}$ promoter region from pKAM312. Figure 4 illustrates the described strategy.

\section{Cultivation in mineral salt medium}

The mineral salt medium used was based on the fermentation medium of Willenbacher et al. [54] with slight changes. The composition of the final medium was: $20 \mathrm{~g} / \mathrm{L}$ glucose, $4.0 \times 10^{-6} \mathrm{M} \mathrm{Na}_{2} \mathrm{EDTA} \times 2 \mathrm{H}_{2} \mathrm{O}$, $7.0 \times 10^{-6} \mathrm{M} \mathrm{CaCl}, 4.0 \times 10^{-6} \mathrm{M} \mathrm{FeSO}+7 \quad \mathrm{H}_{2} \mathrm{O}$, $1.0 \times 10^{-6} \mathrm{M} \mathrm{MnSO} \mathrm{M}_{4} \times \mathrm{H}_{2} \mathrm{O}, 50 \mathrm{mM}$ Urea, $0.03 \mathrm{M}$ $\mathrm{KH}_{2} \mathrm{PO}_{4}, 0.04 \mathrm{M} \mathrm{Na}_{2} \mathrm{HPO}_{4} \times 2 \mathrm{H}_{2} \mathrm{O}$ and $8.0 \times 10^{-4} \mathrm{M}$ $\mathrm{MgSO}_{4} \times 7 \mathrm{H}_{2} \mathrm{O}$.

For the first preculture, $10 \mathrm{~mL} \mathrm{LB}$ medium $(10 \mathrm{~g} / \mathrm{L}$ tryptone, $5 \mathrm{~g} / \mathrm{L} \mathrm{NaCl}, 5 \mathrm{~g} / \mathrm{L}$ yeast extract) was inoculated with $10 \mu \mathrm{L}$ glycerol stock solution in a $100 \mathrm{~mL}$ 


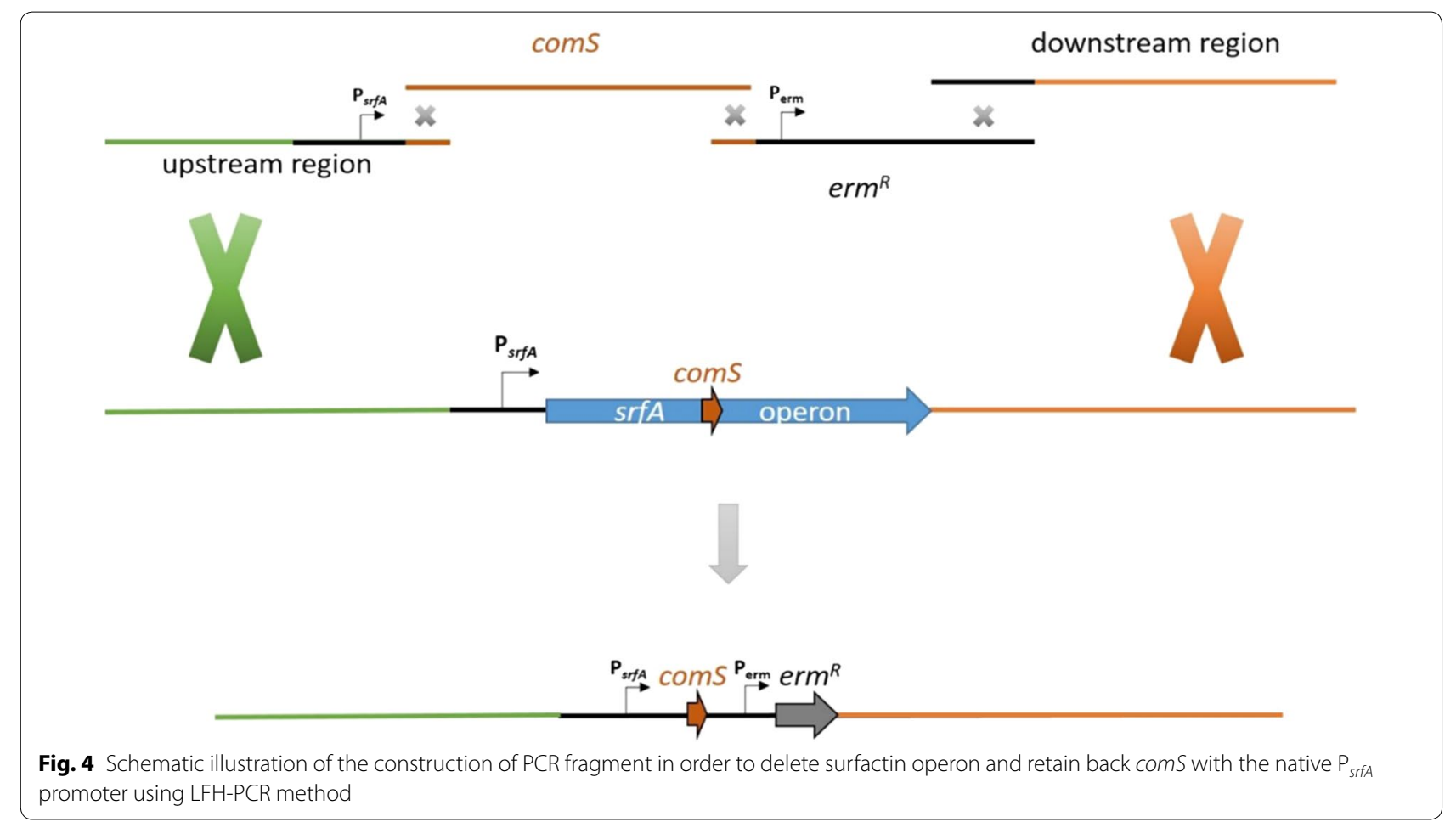

baffled shake flask. After $8 \mathrm{~h}$ of cultivation, the cells were transferred to $10 \mathrm{~mL}$ mineral salt medium with an initial $\mathrm{OD}_{600}$ of 0.1 as a second preculture. This preculture was incubated overnight and after reaching an $\mathrm{OD}_{600}$ between 2-4 the main culture was inoculated. The main cultivations took place in $1 \mathrm{~L}$ Erlenmeyer baffled flasks with the final volume of $100 \mathrm{~mL}$ and initial $\mathrm{OD}_{600}$ of 0.1 . All cultivation had three biological replicates and were performed at $30^{\circ} \mathrm{C}$ and $120 \mathrm{rpm}$ in an incubation shaker (Innova $44^{\circledR} \mathrm{R}$, Eppendorf AG, Hamburg, Germany).

Additionally, the influence of potentially critical amino acids including glutamic acid, glutamine, isoleucine, alanine, proline and ornithine on plipastatin production was tested using mineral salt medium complemented with $30 \mathrm{mM}$ of each amino acids.

\section{Extraction of lipopeptides and HPTLC analysis}

The cell-free supernatants were obtained by centrifugation at $4700 \mathrm{rpm}$ and $4{ }^{\circ} \mathrm{C}$ and were used for extraction of lipopeptides following the method described before with slight changes [55]. In detail, $2 \mathrm{~mL}$ of cell-free supernatant was mixed 3 times with $1 \mathrm{~mL}$ 1-butanol 95\% $(v / v)$ by vortexing for $1 \mathrm{~min}$, followed by $5 \mathrm{~min}$ centrifugation at $3000 \mathrm{rpm}$ to separate organic phase. After complete evaporation of butanol phases (RVC2-25 Cdplus, Martin Christ Gefriertrocknungsanlagen $\mathrm{GmbH}$, Osterode am Harz, Germany) at 10 mbar and $60^{\circ} \mathrm{C}$, the remaining residues were dissolved in $2 \mathrm{~mL}$ methanol. To quantify surfactin and plipastatin production, these methanolic fractions were separated by HPTLC (CAMAG, Muttenz, Switzerland) as described previously [56].

\section{Structural analysis of plipastatin variants by Mass spectrometry}

LC-MS analysis of plipastatin was performed on a 1290 UHPLC system (Agilent, Waldbronn, Germany) coupled to a Q-Exactive Plus Orbitrap mass spectrometer equipped with a heated electrospray ionization (HESI) source (Thermo Fisher Scientific, Bremen, Germany). Analyte separation was achieved by a Waters ACQUITY CSH C18 column $(1.7 \mu \mathrm{m}, 2.1 \mu \mathrm{m} \times 150 \mathrm{~mm})$. The column temperature was maintained at $40{ }^{\circ} \mathrm{C}$. Samples were dissolved in methanol and $5 \mu \mathrm{l}$ of each sample was injected. Mobile phase A was $0.1 \%$ formic acid in water $(\mathrm{v} / \mathrm{v})$, and mobile phase B $0.1 \%$ formic acid in acetonitrile $(\mathrm{v} / \mathrm{v})$. A constant flow rate of $0.3 \mathrm{~mL} / \mathrm{min}$ was used and the gradient elution was performed as follows: 0 $15 \%$ B from 0 to $15 \mathrm{~min}, 15-75 \%$ B from 15 to $29 \mathrm{~min}$, $75-100 \%$ B from 29 to $32 \mathrm{~min}$, isocratic at $100 \%$ B from 32 to $36 \mathrm{~min}$, the system was returned to initial conditions from $100 \%$ B to $0 \%$ B from 36 to $37 \mathrm{~min}$. The HESI source was operated both in positive and negative mode, with a capillary voltage of $3.90 \mathrm{kV}$ and an ion transfer capillary temperature of $350{ }^{\circ} \mathrm{C}$. The sweep 
gas and auxiliary pressure rates were set to 35 and 10, respectively. The S-Lens RF level was $50 \%$, and the auxiliary gas heater temperature was $150{ }^{\circ} \mathrm{C}$. The temperature of ion transfer capillary, spray voltage, sheath gas flow rate, auxiliary gas flow rate and S-lens RF level were set to $325{ }^{\circ} \mathrm{C}, 3.5 \mathrm{kV}, 60,30$ and 55 , respectively. The Q-Exactive Plus mass spectrometer was calibrated externally in positive and negative ion mode using the manufacturer's calibration solutions (Pierce/Thermo Fisher, Germany). Mass spectra were acquired in MS mode within the mass range of $600-1800 \mathrm{~m} / \mathrm{z}$ at a resolution of 70,000 FWHM using an Automatic Gain Control (AGC) target of $1.0 \times 10^{6}$ of and $100 \mathrm{~ms}$ maximum ion injection time. Data dependent MS/MS spectra in a mass range of $200-2000 \mathrm{~m} / \mathrm{z}$ were generated for the five most abundant precursor ions with a resolution of 17,500 FWHM using an Automatic Gain Control (AGC) target of $5.0 \times 10^{4}$ of and $64 \mathrm{~ms}$ maximum ion injection time and a stepped collision energy of 20, 60 and 150. Xcalibur ${ }^{\mathrm{TM}}$ software version 4.0.27 (Thermo Fisher Scientific, San Jose, USA) was used for data acquisition and data analysis.

\section{Supplementary information}

Supplementary information accompanies this paper at https://doi. org/10.1186/s12934-020-01468-0.

Additional file 1. Overview about production of surfactin and corresponding optical densities $\left(\mathrm{OD}_{600}\right)$ of B. subtilis BMV9 $(3 \mathrm{NA} s f p+)$ after $48 \mathrm{~h}$ cultivation in mineral salt medium supplemented with $30 \mathrm{mM}$ of different amino acids.

Additional file 2. A: Plipastatin variants detected by MS analysis in $B$. subtilis standard. B: Detailed mass spectrometry data of extracted-ion chromatograms regarding to plipastatin standard produced by B. subtilis and the sample extracts of BMV12 strain from exponential phase and stationary phase.

\section{Acknowledgments}

We are very grateful to Dr. Josef Altenbuchner for providing strain B. subtilis JABs32.

\section{Authors' contributions}

MV, AR and JP performed the practical work for this study. All authors were involved for interpretation of the results. MV and LL structured the work packages of this project. MV, LL, JP, MH, KMH and RH were involved in writing and editing the manuscript. All authors read and approved the final manuscript.

\section{Funding}

Open Access funding enabled and organized by Projekt DEAL... This work was supported by a grant from the Food Security Center (FSC), an excellence center for exchange and development at the University of Hohenheim (funding reference DAAD 57160040).

\section{Availability of data and materials}

All raw data and biological material are saved in the institute of Food Science and Biotechnology, Department of Bioprocess Engineering (150 k), University of Hohenheim, Fruwirthstraße 12, Stuttgart 70599, Germany. In case of requirement, please contact the corresponding author for any detailed question.
Ethics approval and consent to participate Not applicable.

\section{Consent for publication}

We agree to publish the manuscript and supplemental data.

\section{Competing interests}

Not applicable.

\section{Author details}

${ }^{1}$ Institute of Food Science and Biotechnology, Department of Bioprocess Engineering (150K), University of Hohenheim, Fruwirthstraße 12, 70599 Stuttgart, Germany. ${ }^{2}$ Core Facility Hohenheim, Mass Spectrometry Unit, University of Hohenheim, August-von-Hartmann-Str. 3, 70599 Stuttgart, Germany.

Received: 26 June 2020 Accepted: 29 October 2020

Published online: 10 November 2020

\section{References}

1. van Dijl J, Hecker M. Bacillus subtilis: from soil bacterium to super-secreting cell factory. Microb Cell Fact. 2013;12:3

2. Caulier S, Nannan C, Gillis A, Licciardi F, Bragard C, Mahillon J. Overview of the antimicrobial compounds produced by members of the Bacillus subtilis group. Front Microbiol. 2019;10:302.

3. Vahidinasab M, Ahmadzadeh M, Henkel M, Hausmann R, Heravi KM. Bacillus velezensis UTB96 Is an Antifungal Soil Isolate with a Reduced Genome Size Compared to That of Bacillus velezensis FZB42. Microbiol Res Announc. 2019;8:e00667-e719.

4. Cochrane SA, Vederas JC. Lipopeptides from Bacillus and Paenibacillus spp.: a gold mine of antibiotic candidates. Med Res Rev. 2016;36:4-31.

5. Geissler M, Heravi KM, Henkel M, Hausmann R. Lipopeptide biosurfactants from Bacillus species. In: Hayes D, Solaiman D, Ashby R, editors. Biobased surfactants. New York: Elsevier; 2019. p. 205-40.

6. Raaijmakers JM, de Bruijn I, Nybroe O, Ongena M. Natural functions of lipopeptides from Bacillus and Pseudomonas: more than surfactants and antibiotics. FEMS Microbiol Rev. 2010;34:1037-62.

7. Jacques P. Surfactin and other lipopeptides from Bacillus spp. In: SoberonChavez G, editor. Biosurfactants. Berlin: Springer; 2011. p. 57-91.

8. Cawoy H, Debois D, Franzil L, de Pauw E, Thonart P, Ongena M. Lipopeptides as main ingredients for inhibition of fungal phytopathogens by Bacillus subtilis/amyloliquefaciens. Microb Biotechnol. 2015;8:281-95.

9. Huang X, Lu Z, Zhao H, Bie X, Lü F, Yang S. Antiviral activity of antimicrobial lipopeptide from Bacillus subtilis fmbj against pseudorabies virus, porcine parvovirus, newcastle disease virus and infectious bursal disease virus in vitro. Int J Pept Res Ther. 2006;12:373-7.

10. Yin $H$, Guo C, Wang Y, Liu D, Lv Y, Lv F, Lu Z. Fengycin inhibits the growth of the human lung cancer cell line 95D through reactive oxygen species production and mitochondria-dependent apoptosis. Anticancer Drugs. 2013;24:587-98.

11. Ongena M, Jourdan E, Adam A, Paquot M, Brans A, Joris B, et al. Surfactin and fengycin lipopeptides of Bacillus subtilis as elicitors of induced systemic resistance in plants. Environ Microbiol. 2007;9:1084-90.

12 Hamdache A, Azarken R, Lamarti A, Aleu J, Collado IG. Comparative genome analysis of Bacillus spp. and its relationship with bioactive nonribosomal peptide production. Phytochem Rev. 2013;12:685-716.

13. Inès $M$, Dhouha $G$. Lipopeptide surfactants: production, recovery and pore forming capacity. Peptides. 2015;71:100-12.

14. Wu C-Y, Chen C-L, Lee Y-H, Cheng Y-C, Wu Y-C, Shu H-Y, et al. Nonribosomal synthesis of fengycin on an enzyme complex formed by fengycin synthetases. J Biol Chem. 2007;282:5608-16.

15. Gao L, Guo J, Fan Y, Ma Z, Lu Z, Zhang C, et al. Module and individual domain deletions of NRPS to produce plipastatin derivatives in Bacillus subtilis. Microb Cell Fact. 2018;17:1-13.

16. Hussein W. Fengycin or plipastatin? A confusing question in Bacilli. BioTechnologia. 2019;100:47-55.

17. Samel SA, Wagner B, Marahiel MA, Essen L-O. The thioesterase domain of the fengycin biosynthesis cluster: a structural base for the macrocyclization of a non-ribosomal lipopeptide. J Mol Biol. 2006;359:876-89. 
18. Lambalot RH, Gehring AM, Flugel RS, Zuber P, LaCelle M, Marahiel MA, et al. A new enzyme superfamily - the phosphopantetheinyl transferases. Chem Biol. 1996;3:923-36.

19. Zeigler DR, Prágai Z, Rodriguez S, Chevreux B, Muffler A, Albert T, et al. The origins of 168, W23, and other Bacillus subtilis legacy strains. J Bacteriol. 2008; 190:6983-95.

20 Kunst F, Ogasawara N, Moszer I, Albertini A, Alloni GO, Azevedo V, et al. The complete genome sequence of the gram-positive bacterium Bacillus subtilis. Nature. 1997:390:249-56.

21 Reuß DR, Schuldes J, Daniel R, Altenbuchner J. Complete genome sequence of Bacillus subtilis subsp. subtilis strain 3NA. Genome Announc. 2015:3:e00084-15.

22. Nakano MM, Corbell N, Besson J, Zuber P. Isolation and characterization of $s f p$ : a gene that functions in the production of the lipopeptide biosurfactant, surfactin, in Bacillus subtilis. Mol General Genet MGG. 1992:232:313-21.

23. Tsuge K, Ano T, Hirai M, Nakamura Y, Shoda M. The genes degQ, pps, and Ipa-8 (sfp) are responsible for conversion of Bacillus subtilis 168 to plipastatin production. Antimicrob Agents Chemother. 1999;43:2183-92.

24. Kim PI, Ryu J, Kim YH, Chi Y-T. Production of biosurfactant lipopeptides Iturin A, fengycin and surfactin A from Bacillus subtilis CMB32 for control of Colletotrichum gloeosporioides. J Microbiol Biotechnol. 2010;20:138-45.

25. Yaseen Y, Diop A, Gancel F, Béchet M, Jacques P, Drider D. Polynucleotide phosphorylase is involved in the control of lipopeptide fengycin production in Bacillus subtilis. Arch Microbiol. 2018;200:783-91.

26 Karata AY, Çetin S, Özcengiz G. The effects of insertional mutations in comQ, comP, srfA, spoOH, spoOA and abrB genes on bacilysin biosynthesis in Bacillus subtilis. Biochimica et Biophysica Acta BBA. 2003;1626:51-6.

27. Zeriouh $H$, de Vicente A, Pérez-García A, Romero D. Surfactin triggers biofilm formation of Bacillus subtilis in melon phylloplane and contributes to the biocontrol activity. Environ Microbiol. 2014:16:2196-211.

28. Chumsakul O, Takahashi H, Oshima T, Hishimoto T, Kanaya S, Ogasawara N, Ishikawa S. Genome-wide binding profiles of the Bacillus subtilis transition state regulator $\mathrm{AbrB}$ and its homolog $\mathrm{Abh}$ reveals their interactive role in transcriptional regulation. Nucleic Acids Res. 2011:39:414-28.

29. Do T-H, Suzuki Y, Abe N, Kaneko J, Itoh Y, Kimura K. Mutations suppressing the loss of DegQ function in Bacillus subtilis (natto) poly- - -glutamate synthesis. Appl Environ Microbiol. 2011;77:8249-58.

30. Tsuge K, Matsui K, Itaya M. Production of the non-ribosomal peptide plipastatin in Bacillus subtilis regulated by three relevant gene blocks assembled in a single movable DNA segment. J Biotechnol. 2007;129:592-603.

31. Wang P, Guo Q, Ma Y, Li S, Lu X, Zhang X, Ma P. DegQ regulates the production of fengycins and biofilm formation of the biocontrol agent Bacillus subtilis NCD-2. Microbiol Res. 2015;178:42-50.

32. Stanley NR, Lazazzera BA. Defining the genetic differences between wild and domestic strains of Bacillus subtilis that affect poly- $\gamma$-DL-glutamic acid production and biofilm formation. Mol Microbiol. 2005;57:1143-58.

33. Kaki AA, Smargiasso N, Ongena M, Ali MK, Moula N, de Pauw E, Chaouche NK. Characterization of New Fengycin Cyclic Lipopeptide variants produced by Bacillus amyloliquefaciens (ET) originating from a Salt Lake of Eastern Algeria. Curr Microbiol. 2020;77:443-51.

34. Li X-Y, Mao Z-C, Wang Y-H, WU Y-X, He Y-Q, Long C-L. ESI LC-MS and MS/ MS characterization of antifungal cyclic lipopeptides produced by Bacillus subtilis XF-1. J Mol Microbiol Biotechnol. 2012;22:83-93.

35. Miras M, Dubnau D. A DegU-P and DegQ-dependent regulatory pathway for the K-state in Bacillus subtilis. Frontiers in microbiology. 2016;7:1868.

36. Jeong D-E, So Y, Lim H, Park S-H, Choi S-K. Scarless genomic point mutation to construct a Bacillus subtilis strain displaying increased antibiotic plipastatin production. J Microbiol Biotechnol. 2018;28:1030-6.

37. Yaseen $Y$, Gancel F, Drider D, Béchet M, Jacques P. Influence of promoters on the production of fengycin in Bacillus spp. Res Microbiol. 2016:167:272-81.
38. Mäder U, Antelmann H, Buder T, Dahl M, Hecker M, Homuth G. Bacillus subtilis functional genomics: genome-wide analysis of the DegS-DegU regulon by transcriptomics and proteomics. Mol Genet Genomics. 2002;268:455-67.

39. López D, Kolter R. Extracellular signals that define distinct and coexisting cell fates in Bacillus subtilis. FEMS Microbiol Rev. 2010;34:134-49.

40. Briani F, Carzaniga T, Dehò G. Regulation and functions of bacterial PNPase. Wiley Interdiscipl Rev RNA. 2016;7:241-58.

41. Yaseen Y, Gancel F, Béchet M, Drider D, Jacques P. Study of the correlation between fengycin promoter expression and its production by Bacillus subtilis under different culture conditions and the impact on surfactin production. Arch Microbiol. 2017;199:1371-82.

42. Geiger T, Wolz C. Intersection of the stringent response and the CodY regulon in low GC Gram-positive bacteria. Int J Med Microbiol. 2014;304:150-5.

43. Steinchen W, Bange G. The magic dance of the alarmones (p) ppGpp. Mol Microbiol. 2016;101:531-44.

44 Sambrook J, Russell DW, Sambrook J. The condensed protocols: from molecular cloning: a laboratory manual. Cold Spring Harbor, NY: Cold Spring Harbor Laboratory Press; 2006.

45. Chung CT, Niemela SL, Miller RH. One-step preparation of competent Escherichia coli: transformation and storage of bacterial cells in the same solution. Proc Natl Acad Sci. 1989;86:2172-5.

46. Heravi KM, Altenbuchner J. Cross talk among transporters of the phosphoenolpyruvate-dependent phosphotransferase system in Bacillus subtilis. J Bacteriol. 2018;200:e00213-e218.

47. Harwood CR, Cutting SM. Molecular biological methods for Bacillus. Hoboken, NJ: Wiley; 1990

48. Yanisch-Perron C, Vieira J, Messing J, Chambers SP, Prior SE, Barstow DA, et al. Improved M13 phage cloning vectors and host strains: nucleotide. Gene. 1985:33:103-19.

49. Geissler M, Kühle I, Heravi KM, Altenbuchner J, Henkel M, Hausmann R. Evaluation of surfactin synthesis in a genome reduced Bacillus subtilis strain. AMB Express. 2019;9:1-14.

50. Michel JF, Millet J. Physiological Studies on Early-blocked Sporulation Mutants of Bacillus subtilis. J Appl Bacteriol. 1970;33:220-7.

51. Wenzel M, Altenbuchner J. Development of a markerless gene deletion system for Bacillus subtilis based on the mannose phosphoenolpyruvatedependent phosphotransferase system. Microbiology. 2015;161:1942-9.

52. Altenbuchner J, Viell P, Pelletier I. [40] Positive selection vectors based on palindromic DNA sequences. Methods Enzymol. 1992;216:457-66.

53 Wach A. PCR-synthesis of marker cassettes with long flanking homology regions for gene disruptions in S. cerevisiae. Yeast. 1996;12:259-65.

54. Willenbacher J, Zwick M, Mohr T, Schmid F, Syldatk C, Hausmann R. Evaluation of different Bacillus strains in respect of their ability to produce Surfactin in a model fermentation process with integrated foam fractionation. Appl Microbiol Biotechnol. 2014:98:9623-32.

55 Yazgan A, Özcengiz G, Marahiel MA. Tn10 insertional mutations of Bacillus subtilis that block the biosynthesis of bacilysin. Biochem Biophys Acta. 2001;1518:87-94.

56. Geissler M, Oellig C, Moss K, Schwack W, Henkel M, Hausmann R. Highperformance thin-layer chromatography (HPTLC) for the simultaneous quantification of the cyclic lipopeptides surfactin, iturin $A$ and fengycin in culture samples of Bacillus species. J Chromatogr B. 2017;1044:214-24.

\section{Publisher's Note}

Springer Nature remains neutral with regard to jurisdictional claims in published maps and institutional affiliations. 